\title{
Análisis cuantitativo de la influencia de algunos Factores de Riesgo Gineco-Obstétrico*
}

\author{
Dres.: Heliodoro Ñáñez Burbano y Wayne Montoya Guerrero*
}

\section{INTRODUCCION}

El conocimiento de las altas cifras de morbi-mortalidad materna y fetal en los países en desarrollo, ha generado a nivel de gobiernos y organizaciones de salud, nuevas políticas y asignación de mayores recursos a la investigación de los factores condicionantes y determinantes del riesgo obstétrico, con el fin de obtener bases firmes para el diagnóstico y control de los factores de riesgo que puedan alterar el desarrollo normal del parto y la salud, tanto de la madre como del recién nacido.

En Colombia, de acuerdo con el plan diseñado para la atención hospitalaria en el Sistema Nacional de Salud, al presentar la gestante alguno o algunos de los factores de riesgo se le clasifica como de riesgo alto, medio o bajo y de acuerdo con esta clasificación se atiende su emba-

* Realizado en el Hospital Materno Infantil de Bogotá. D.E.

** Estadístico. razo, parto y puerperio, en hospitales de nivel alto, regionales o centros de salud.

Entre los principales factores de riesgo obstétrico son conocidos: los antecedentes gineco-obstétricos, las características que presenta el embarazo actual, la patología materna agregada, los factores. de riesgo intraparto y la situación socioeconómica.

Teniendo en cuenta la importancia que representa para el hospital el conocimiento de los factores de riesgo obstétrico, este trabajo pretende identificar, clasificar y jerarquizar los diferentes factores determinantes de riesgo mediante la discriminación de los factores en forma aislada $y$ en conjunto para diferenciar a las gestantes con al to o bajo riesgo y también para explorar la existencia de interrelaciones entre los factores de riesgo obstétrico.

\section{OBJETIVO GENERAL}

Realizar análisis cuantitativo de factores determinantes y condicionantes de riesgo obstétrico in trahospitalario. 


\section{Objetivos Específicos}

1. Identificar los factores que más influyen en el riesgo obstétrico intrahospitalario de los considerados para el presente estudio.

2. Cuantificar el aporte de cada uno de los factores al riesgo obstétrico intrahospitalario.

3. Explorar la factibilidad de construir una clasificación de riesgo obstétrico intrahospitalario con base en la importancia de cada uno de los factores de riesgo.

4. Explorar la existencia de interrelaciones dentro del conjunto de factores de riesgo obstétrico.

\section{Diseño de la Muestra e Instrumento de Recolección}

Para obtener la información pertinente $y$ así poder abordar el problema objeto del estudio, se diseñó una muestra sistemática de historias clínicas de pacientes que dieron a luz en el Hospital Materno Infantil durante el año de 1984 -estudio retrospectivo-. Con el fin de recolectar la información se elaboró un primer formulario, el cual fue puesto a prueba, mediante una muestra piloto de 60 historias clínicas, proceso que permitió visualizar algunos inconvenientes como: no existencia de historias seleccionadas, historias incompletas.

De acuerdo con lo anterior, se rediseñó la muestra y un instrumento para la recolección.

El tamaño de la muestra se determinó teniendo en cuenta entre otros aspectos, que todas las variables en estudio son dicotómicas o fueron dicotomizadas. Este hecho hizo que se utilizarà la expresión usual para estimar el tamaño de muestra, cuando se pretende valuar proporciones. También, debido a que se trata de una visión prospectiva del fenómeno, esto obliga a que se utilice la expresión para poblaciones infinitas:

$$
n=\frac{Z^{\alpha} / 2 P(1-P)}{e^{2}}
$$

Como es sabido esta expresión para $n$ se hace máxima tomando $P=0,5$; con esta consideración $y$ teniendo en cuenta que se escogió $1-\alpha=0,95$-nivel de confianza- y e $=0,05$-máximo error permisible - la estimación del tamaño de muestra fue de 385 .

El instrumento que se utilizó para la recolección de la información fue un formulario teniendo en cuenta el replanteamiento del trabajo.

\section{Recolección de la Información}

Teniendo en cuenta que el período de recolección de la información se había programado del 11 de junio al 10 de julio de 1985 , o sea un mes, y que el Hospital atiende en promedio, aproximadamente 1.000 partos al mes, se vio la oportunidad de aumentar el tamaño de muestra, previendo $\mathrm{mal}$ diligenciamiento de los formularios u otros aspectos que alterarían en parte la calidad de la información y además, para allegar mayor información sobre las variables incluidas en el estudio.

De los 1.000 formularios entregados se recibieron 541, de los cuales fueron rechazados 52 por las siguientes causas: partos gemelares (9) -doble información para cada uno de estos en la parte concerniente al recién nacido-, abortos (20), 
manejo inadecuado del parto extrahospitalario (5), embarazos ectópicos o tubáricos (5) -de estos treinta formularios no se tiene resultado final del recién nacido- y 13 formularios que no tenían la información completa y además, no se encontró la historia clínica.

Después de este proceso, el número de formularios se redujo a 489, número que se utiliza para el presente trabajo.

\section{Depuración de la Información}

La crítica de los formularios en cuanto a la parte correspondiente a los conceptos gineco-obstétricos fue realizada en su totalidad por el doctor Arturo Rodríguez Soto, quien verificó la consistencia interna que presentaba la información recolectada.

Terminada la primera etapa manual de crítica de la información contenida en los formularios, se procedió a partir del 30 de septiembre de 1985, a codificar la información para posteriormente ser sometida a procesamiento.

Este procesamiento se dividió en dos etapas a saber: la primera de ellas consistió básicamente en la obtención de distribuciones de frecuencia, etapa que permitió posteriormente dicotomizar algunas variables e identificar aquellas de varianza idénticamente igual a cero, para determinar finalmente el grupo de variables.

La segunda etapa del procesamiento se dedicó a la aplicación de procedimientos implementados en SAS y SPSS, procedimientos pertinentes para el logro de los objetivos propuestos.

\section{Clasificación de Variables}

Las variables en estudio, con propósitos de análisis se clasifican en dos grupos:
1. Variables consideradas como daño y/o complicación.

2. Variables consideradas como posibles factores de riesgo.

Según Renate Plaut, se define daño, como: "la aparición o existencia de un proceso patológico o de complicaciones de ese proceso". Según esta definición se consideraron las siguientes variables como daño, ya que son patología o son complicaciones, en el proceso de parto:

embarazo prolongado

macrosomía fetal

muerte fetal in útero

presentaciones distócicas incluyendo pelvis

hemorragia genital

desproporción céfalo-pélvica

procúbito o procidencia del cordón

sufrimiento fetal agudo

sufrimiento fetal crónico

manejo inadecuado extrahospitalario del parto

infección amniótica

distocias mecánicas

distocias dinámicas

ruptura uterina

traumatismo*

desprendimiento placenta

placenta previa

parto prematuro

presentación del feto en podálica o situación transversa

parto intervenido

alumbramiento patológico

recién nacido muerto

peso mayor de 4.000 gramos o menor de 2.500 gramos

apgar menor o igual a 5

síndrome de dificultad respiratoria del recién nacido

* no se presentó 


\author{
patología neurológica \\ anomalías congénitas mayores \\ isoinmunización \\ hiperbilirrubinemia \\ infección \\ prematurez o bajo peso al nacer \\ post-madurez \\ hemorragia cerebral* \\ trauma obstétrico \\ recién nacido con complicaciones \\ puerperio patológico \\ madre con complicaciones
}

Renate Plaut (20), define el riesgo como: "la probabilidad de que ocurra un fenómeno indeseado o daño" y factor de riesgo como: "todo factor, característica o circunstancia que va acompañada de un aumento de la probabilidad o riesgo de que ese daño ocurra, sin prejuzgar si el factor en cuestión es o no una de las causas del daño, aun cuando, como ocurre por lo general, su identificación haya sido motivada por una sospecha de causalidad". De acuerdo con lo anterior, a continuación se presentan las variables que fueron determinadas como posibles factores de riesgo:

aborto habitual*
paridad mayor de 5
muertes perinatales
cesáreas previas
cirugía uterina o vaginal
anomalías congénitas mayores
recién nacido macrosómico
recién nacido de bajo peso
menor de 15 años
peso menor de 40 kilogramos*
peso mayor de 75 kilogramos
talla menor de 1,45 centímetros
primigestante mayor de 30 años
anomal ías genitourinarias
incompetencia cervical

* no se presentaron

\author{
tumores genitales* \\ cardiopatías \\ neumopatías \\ hepatopatías \\ neuropatías \\ endocrinopatíds \\ psicopatías* \\ nefropatías \\ enfermedades infecciosas o parasitarias \\ enfermedad vascular hipertensiva cró- \\ nica \\ cáncer \\ discracias sanguíneas \\ varices \\ anemia con hemoglobina menor de 9 \\ gramos \\ toxemia gravídica \\ irradiación o tóxicos* \\ madre RH negativa \\ retardo de crecimiento intrauterino \\ anomalías pelvis \\ polihidramnios \\ rup tura prematura de membranas
}

Como puede verse de los posibles 37 factores de riesgo, se excluyen del análisis 7 de ellos, quedando de esta manera 30.

\section{Selección de Factores de Riesgo}

La selección de los factores de riesgo se realizó teniendo en cuenta el grado de daño y/o complic ación que se presentó.

Se creó la variable "daño global" dicotómica de la siguiente manera:

0: cuando en el proceso de parto no se presentó ninguno de los daños $y / o$ complicaciones anteriormente mencionados.

1: cuando en el proceso de parto se presentó por lo menos un daño y/o la complicación. 
Es así como de las 489 pacientes seleccionadas para el trabajo, 224 de ellas no presentaron algún daño y/o la complicación en el proceso de parto y el resto presentaron por lo menos algún daño $y / 0$ complicación. Su distribución con respecto al número de factores de riesgo presentados por las pacientes, aparecen en la Tabla No. 1.

Utilizando el estimador de máxima verosimilitud para la probabilidad de éxito en una distribución de Bernoulli Y' con la información de la Tabla No. 1, el riesgo de presentar daño y/o complicación durante el proceso de parto, se estima en 0,5419 .

Con el fin de seleccionar los factores de riesgo y de jerarquizar la importancia de cada uno de ellos, se utilizaron como criterios el riesgo relativo y la prueba ji-cuadrado para independencia de atributos de la siguiente manera:

TABLA No. 1

\section{DISTRIBUCION DE LAS PACIENTES DE ACUERDO CON EL NUMERO DE FACTORES DE RIESGO Y LA EXISTENCIA O NO DE DAÑO Y/O COMPLICACION}

\begin{tabular}{|c|c|c|c|c|c|c|c|c|}
\hline \multirow{1}{*}{$\begin{array}{c}\text { Daxa y/0 } \\
\text { complicacion }\end{array}$} & No & 90 & 102 & 24 & 6 & 2 & 0 & 224 \\
\cline { 2 - 9 } & Si & 79 & 119 & 48 & 14 & 4 & 1 & 265 \\
\hline Total & 169 & 221 & 72 & 20 & 6 & 1 & 489 \\
\hline
\end{tabular}

\section{Riesgo Relativo}

En la Tabla No. 2, aparece el valor de riesgo relativo, se puede ver que 22 factores de riesgo tuvieron un valor de riesgo relativo mayor que la unidad, de lo cual se puede deducir que estos factores de riesgo pueden estar implicados como responsables de un daño $y / o$ complic ación.

Los factores que presentaron los más altos valores de riesgo relativo fueron: antecedentes de muertes perinatales $(1,87160)$, siguiéndole cirugía uterina o vaginal previa $(1,8648)$ y recién nacidos macrosómicos $(1,8582)$, siguen $5 \mathrm{fac}$ tores de riesgo con riesgo relativo de 1,85171, los cuales son: cardiopatías, neumopatías, anomalías pelvis, polihidramnios y endocrinopatías.

\section{Prueba para Independencia}

Con el fin de valorar la significancia del riesgo relativo se utilizó la prueba para in- 
TABLA No. 2

\section{VALORES DE RIESGO RELATIVO, JERARQUIA, JI-CUADRADO, SIGNIFICANCIA Y JERARQUIA DE LOS FACTORES}

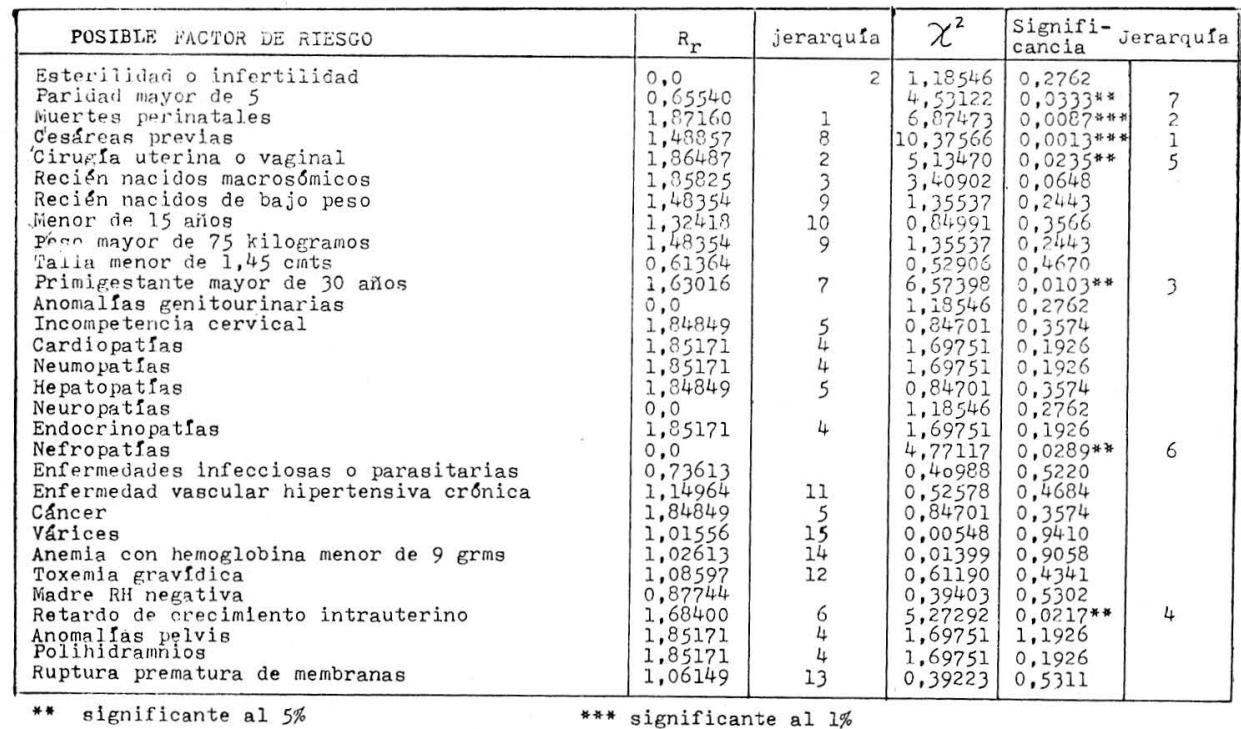

dependencia en tablas de contingencia $2 \times 2$, basada en la distribución jicuadrado.

A través de esta prueba se puede cuantificar la asociación estadística entre la variable daño global (dicotómica) y cada posible factor de riesgo (dicotómico) y así poder seleccionar un subconjunto de factores de riesgo.

En la Tabla No. 2, se presentan los valores de "ji-cuadrado", su significancia y la jerarquía desde el punto de vista de su magnitud. En la última columna se puede observar la jerarquización de los factores de riesgo de acuerdo con el grado de asociación.

\section{Selección y Jerarquización}

La selección de los factores de riesgo utilizando el riesgo relativo, condujo a obtener 22 factores que presentan riesgo relativo mayor de la unidad y por medio de la ji-cuadrado para independencia, se obtuvieron siete factores de riesgo asociados significativamente con el daño global, a un nivel de confianza del $95 \%$. A continuación se presenta la Tabla No. 3 con los valores de riesgo relativo y jicuadrado calculado:

En la tabla aparecen siete factores, de riesgo seleccionados mediante la prueba ji-cuadrado. Además se puede observar que aparecen dos factores de riesgo: nefropatías y paridad mayor de 5, que tuvieron un riesgo relativo menor que la unidad.

La úl tima selección se realizó tomando aquellos factores que resultaron significativos al $5 \%$ y riesgo relativo mayor de 
Primigestante mayor de 30 años 1,63016

Retardo de crecimiento intrauterino

1,68400

5,27292

Cirugia uterina o vagina!

1,86487

5,13470

Nefropatias

0,0

$4,771 ! 7$

Paridad mayor de 5

0,65540

4,53122

la unidad y su jerarquización se hizo teniendo en cuenta, el valor calculado de la ji-cuadrado, esto condujo a seleccionarlos y jerarquizarlos en el siguiente orden:

\section{Cesáreas prevìas}

Muertes perinatales

Primigestante mayor de 30 años

Retardo de crecimiento intrauterino

Cirugía uterina o vaginal

\section{Clasificación de Pacientes}

De la información obtenida sobre los 489 partos, se identificaron 224 sin complicación y/o daño, tanto para la madre como para el recién nacido y los denotamos como pertenecientes al grupo $\xi_{1}$. Los 265 restantes que presentaron complicaciones $y / 0$ daños durante el proceso de parto como $\xi_{2}$. En el numeral anterior se seleccionaron y jerarquizaron 5 factores de riesgo, que a continuación se anotan de la siguiente manera:

$-\mathrm{X}_{1}: \quad$ cesáreas previas

$-x_{2}:$ muertes perinatales

$-X_{3}$ : primigestante mayor de 30 años

$-X_{4}$ : retardo de crecimiento intrauterino

$-\mathrm{X}_{5}: \quad$ cirugía u terina o vaginal

Las distribuciones de frecuencia para los dos grupos con respecto a los $5 \mathrm{fac}$ tores de riesgo, se presentan en la Tabla No. 4 y además, en el diagrama No. 1 .

Como puede observarse de los 32 posibles estados, solamente 9 de ellos presentan frecuencias totales mayores a cero. 
TABLA No. 4

DISTRIBUCION DE PACIENTES DE ACUERDO CON LOS FACTORES DE RIESGO Y GRUPO DE RIESGO

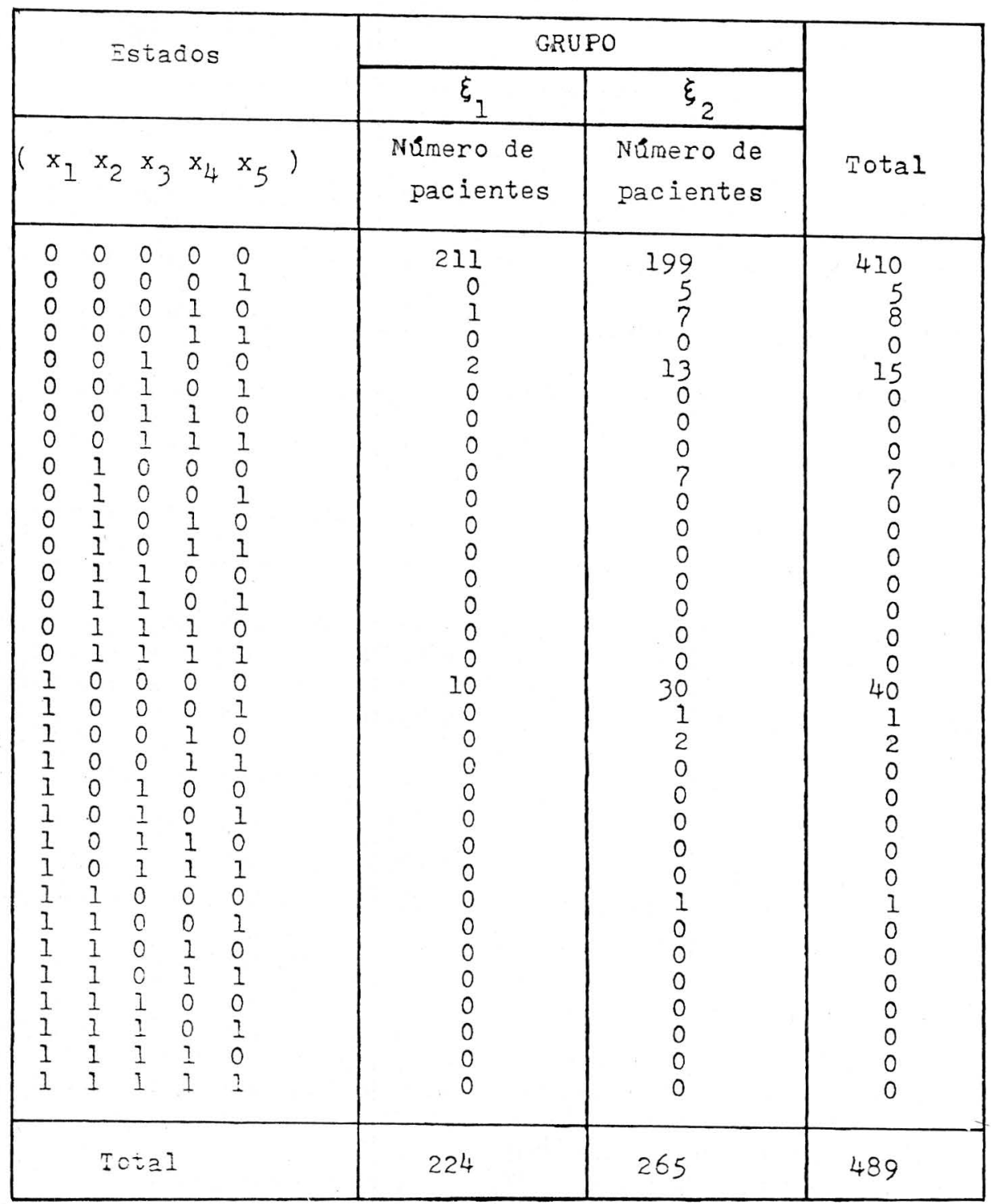


DIAGRAMA No. 1

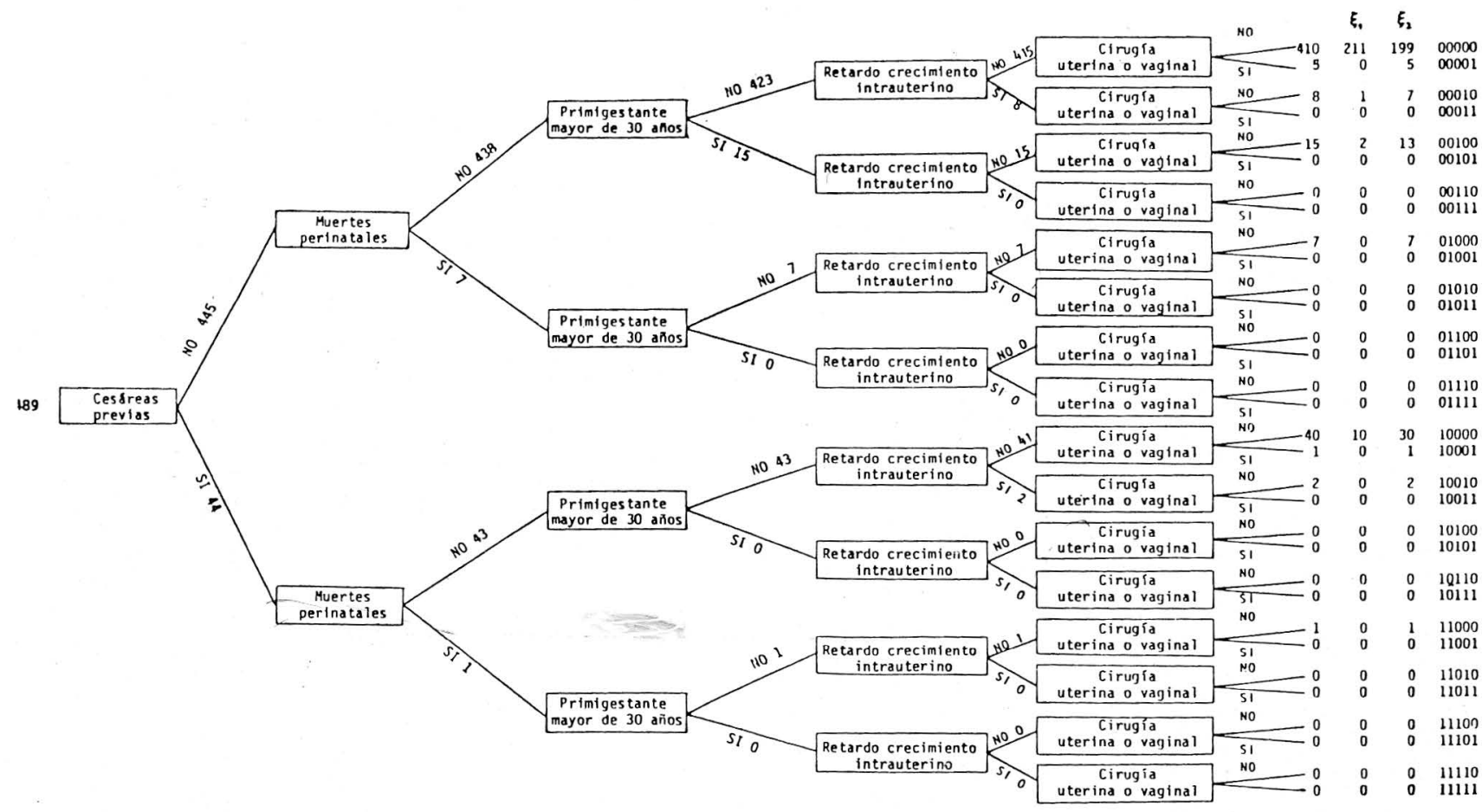


Para clasificar a las pacientes en $\xi 10$ $\xi 2$ se utilizan los métodos: multinomial completo y vecino más cercano de orden $r=1$. El método de clasificación no se usa debido a que en el método asume que todas las variables son independientes y sería poco realista usarlo en éste trabajo.

En las Tablas Nos. 5 y 6 se presentan los resultados del método de clasificación "multinomial completo" y vecino más cercano del orden $r=1$ ".
La clasificación de pacientes haciendo uso del "método multinomial completo", se puede realizar de la siguiente manera:

- Paciente que ingrese al hospital en proceso de parto y no presente ninguno de los cinco factores de riesgo en consideración, será clasificada en el grupo $\xi 1$; es decir, como perteneciente al grupo de "RIESGO BAJO".

- En el grupo $\xi 2$ o perteneciente al grupo de "RIESGO ALTO", sería clasifi-

TABLA No. 5

CLASIFICACION POR EL METODO "MULTINOMIAL COMPLETO”

\begin{tabular}{|c|c|c|c|c|c|c|c|c|c|c|c|c|}
\hline$\left(x_{1}\right.$ & $\begin{array}{l}\text { Est } \\
x_{2}\end{array}$ & $\begin{array}{l}\operatorname{tano} \\
x_{3}\end{array}$ & & $\left.x_{5}\right)$ & $N_{1}(x x)$ & $N_{2}(x x)$ & $f_{1}(x)$ & $f_{2}(x x)$ & $n_{1}(x)$ & $h_{2}(x)$ & Localizacion & $\mathrm{S}^{*}\left(\mathrm{~A}^{*}\right)$ \\
\hline 0 & 0 & 0 & 00 & 0 & 211 & 199 & $0.9^{\prime}+2$ & 0.751 & 0,4314 & 0,4070 & $\xi_{1}$ & 0.4070 \\
\hline 0 & 0 & 0 & $0 \quad 1$ & 1 & 0 & 5 & 0,0 & 0,019 & 0,0 & 0.01 .03 & $\xi_{2}$ & 0.0 \\
\hline 0 & 0 & 0 & 1. 0 & 0 & 1 & 7 & 0.004 & 0,026 & 0,0018 & 0.0141 & $\xi_{2}$ & 0.0018 \\
\hline 0 & 0 & 1 & $0 \quad 0$ & 0 & 2 & 13 & 0.009 & 0.049 & 0,0041 & 0.0266 & $\xi_{2}$ & 0.0042 \\
\hline 0 & 1 & 0 & $0 \quad 0$ & n & 0 & 7 & 0,0 & 0,026 & 0,0 & 0.0141 & $\xi_{2}$ & 0.0 \\
\hline 1 & 0 & 0 & $0 \quad 0$ & 0 & 10 & 30 & 0,045 & 0,113 & 0,0210 & 0,0612 & $\xi_{2}$ & 0,0210 \\
\hline 1 & 0 & 0 & 0 J. & ]. & 0 & 1 & 0,0 & 0,004 & 0,0 & 0,0022 & $\xi_{2}$ & 0,0 \\
\hline 1 & 0 & 0 & 10 & 0 & 0 & 2 & 0,0 & 0.008 & 0,0 & 0,0043 & $\xi_{2}$ & 0,0 \\
\hline 1 & 1 & 0 & 00 & 0 & 0 & 1 & 0,0 & 0,004 & 0.0 & 0,0022 & $\xi_{2}$ & 0,0 \\
\hline & & $\operatorname{tal}$ & & & 224 & 265 & 1,000 & 1,000 & & & & 0,4339 \\
\hline
\end{tabular}

cada la paciente que ingrese al hospital en trabajo de parto y que presente alguno de los cinco factores de riesgo en consideración o cesárea previa acompañada por cualquiera de los factores: cirugía uterina o vaginal, retardo de crecimiento intrauterino o muertes perinatales.
Por medio del método de clasificación, "vecino más cercano de orden $r=1$ ", la paciente que ingrese al hospital en proceso de parto será clasificada en el grupo $\xi 20$ de RIESGO ALTO, ya sea que no presente ninguno de los factores de riesgo considerados o que presente al menos uno de ellos. 
TABLA No. 6

CLASIFICACION POR EL METODO DEL

"VECINO MAS CERCANO DE ORDEN $r=1$ "

\begin{tabular}{|c|c|c|c|c|c|c|c|c|c|c|c|c|c|}
\hline$\left(x_{1}\right.$ & $\begin{array}{l}\text { Estad } \\
x_{2} x_{3}\end{array}$ & & $\left.x_{5}\right)$ & $n_{1}(x)$ & $n_{2}(x)$ & $n_{1}(z)$ & $n_{2}(z)$ & $f_{1}(z)$ & $f_{2}(\mathbb{z})$ & $h_{1}(\mathbb{z})$ & $h_{2}(\mathbb{z})$ & $\begin{array}{l}\text { Locali- } \\
\text { zacion }\end{array}$ & $g^{*}\left(A^{*}\right)$ \\
\hline 0 & 00 & 0 & 0 & 211 & 199 & 224 & 261 & 0,168 & 0,181 & 0,0770 & 0.0981 & $\xi_{2}$ & 0.0770 \\
\hline 0 & 00 & 0 & 1 & 0 & 5 & 211 & 205 & 0,158 & 0,142 & 0,0724 & 0,0770 & $\xi_{2}$ & 0.0724 \\
\hline 0 & 00 & 1 & 0 & 1 & $?$ & 212 & 208 & 0.159 & 0,145 & 0.0728 & 0.0786 & $\xi_{2}$ & 0.0728 \\
\hline 0 & 01 & 0 & 0 & 2 & 13 & 213 & 212 & 0,160 & 0.147 & 0,0733 & 0.0797 & $\xi_{2}$ & 0.0733 \\
\hline 0 & 10 & 0 & 0 & 0 & $?$ & 211 & 207 & 0,158 & 0,144 & 0,0724 & 0,0780 & $\xi_{2}$ & 0,0724 \\
\hline 1 & 00 & 0 & 0 & 10 & 30 & 231 & 233 & 0.173 & 0,162 & 0,0792 & 0,0878 & $\xi_{2}$ & 0,0792 \\
\hline 1 & $0 \quad 0$ & 0 & 1 & 0 & 1 & 10 & 36 & 0.007 & 0.025 & 0,0032 & 0,0136 & $\xi_{2}$ & 0,0032 \\
\hline 1 & $0 \quad 0$ & 1 & 0 & 0 & 2 & 11 & 39 & 0,008 & 0,027 & 0,0037 & 0,0146 & $\xi_{2}$ & 0,0037 \\
\hline 1 & 10 & 0 & 0 & 0 & 1 & 10 & 38 & 0,007 & 0.026 & 0,0032 & 0,0141 & $\xi_{2}$ & 0,0032 \\
\hline & Tota & & & 224 & 265 & 1333 & 1439 & 1,000 & 1,000 & & & & $0.457 c$ \\
\hline
\end{tabular}

El procedimiento multinomial completo difiere del procedimiento del vecino más cercano de orden $r=1$ en la localización para el primer estado, además, la probabilidad de mala clasificación es más al ta $(0,4070)$ para éste estado. En cuanto a las probabilidades de mala clasificación es más al ta cuando se utiliza el procedimiento del vecino más cercano de orden $r=1$.

\section{CONCLUSIONES Y RECOMENDACIONES}

1. Es evidente que la información obtenida para este trabajo, nos permitió llevar a cabo un análisis exhaustivo de riesgo, por medio de algunos procedimientos estadísticos especiales, planeados inicialmente para lograr el objetivo general, pero no hay elementos para afirmar que es deficiente la calidad de la información, pues además de haber impartido instrucciones precisas al personal médico respecto al registro de la información, se realizó un riguroso proceso de depuración, tanto manual como por medio del computador.

La anterior afirmación se deduce al observar la frecuencia con la cual se presentaron los factores de riesgo, en la mayoría de ellos es relativamente baja y aun nula. Esta dificultad puede tener dos causas principales. La prime$\mathrm{ra}$, es que realmente dichas frecuencias sean bajas, es decir, son fenómenos de poca ocurrencia ante lo cual, el rumbo de un próximo trabajo sobre este tema, necesariamente debe ser distinto al seguido por el presente. La segunda causa posible, puede haber radicado en un escaso tamaño de muestra, que aun cuando dicho tamaño fue aumentado cerca de un $27 \%$, no permitió registrar la frecuencia real de algunos factores de riesgo. 
2. Si la causa determinante, fue el haber contado con un tamaño de muestra insuficiente, implica que además de elegir procedimientos diferentes al utilizado (como el uso de alguna función de potencia para determinarlo, o algún procedimiento que pueda derivar el tamaño, controlando errores como el de mala clasificación) necesariamente debe extenderse la observación del fenómeno por un lapso de tiempo superior.

3. Debido al supuesto de que el Hospital Materno Infantil atiende casos de al to riesgo, se espera que los factores de riesgo se presentarán en proporciones mayores a las obtenidas. De hecho, el hospital está cumpliendo funciones más allá de las de atender exclusivamente el llamado alto riesgo y por ende disponiendo de recursos en la atención del parto "normal" que podrían canalizarse hacia los partos con alguna complicación o daño; esta afirmación se basa en que solamente el $54,2 \%$ de los casos presentan daño y/o complicación durante el proceso de parto, cifra considerablemente significativa en el volumen de atención que presta la institución.

4. Como consecuencia de todo lo anterior, tanto la jerarquización de factores, como la clasificación propuesta en este trabajo, deben entenderse solamente de manera tentativa, provisional, hasta tanto no se lleven a cabo estudios similares de mayor cobertura en tiempo y respaldados en recursos financieros y humanos.

5. El hecho de que se presenten pacientes sin ningún factor de riesgo y sufran daño y/o complicación durante el proceso de parto, tanto ellas como los recién nacidos, hace sospechar que están involucrados otros factores de riesgo no considerados en el presente trabajo.

6. El ingreso de pacientes al Hospital y que no sufren de daño y/o complicación durante el proceso de parto, habiendo presentado uno o varios factores de riesgo, hace pensar en el papel importante que juega la intervención médica para modificar el desarrollo de procesos patológicos o de complicaciones.

7. Con el fin de cuantificar el aporte de cada uno de los factores al riesgo obstétrico intrahospitalario, se pensó en utilizar la función logit, pero por lo anotado en la conclusión 1 , no fue posible.

8. El análisis de factores y componentes principales, no permite visualizar una estructura de factores, posiblemente debido al mismo problema de la conclusión 1.

9. Se recomienda hacer el estudio de riesgo obstétrico en forma prospectiva y utilizando un formulario adecuado, debido a que las historias clínicas no presentan la información requerida.

10. Al estudiar el riesgo de un daño $y / 0$ complicación en particular o un conjunto de ellos, se recomienda estudiar aquellos casos de los cuales se pueda obtener suficiente información en consulta prenatal, esto es, no considerar aquellos que carezcan de por lo menos un número a especificar de controles prenatales, ésto con el fin de desarroIlar un análisis más amplio con información suficiente del desarrollo del embarazo; además, hacer un segui- 
miento del recién nacido con el fin de establecer posibles daños y/o complicaciones que no se presentaron durante el período de estancia en el hospital.

\section{RESUMEN}

El estudio se realizó en el Hospital Materno Infantil de Bogotá D.E. con el fin de conocer los factores de riesgo obstétrico intrahospitalario. Se analizaron prospectivamente 489 partos. Se seleccionaron y jerarquizaron 5 factores de riesgo (de 30 posibles) asociados significativamente con el daño y/o complicación y riesgo relativo mayor de la unidad. Se clasifican tentativamente las pacientes con riesgo alto o bajo utilizando los métodos multinomial completo y vecino más cercano de orden $r=1$. Se realizó

\section{BIBLIOGRAFIA}

1. AGUERO, OSCAR. Alto riesgo obstétrico: Rev. Obst. y Gin. Venezuela (Caracas). 41 (4): 197-210, jul., 1981.

2. AUBRY, RICHARD y NESBITT, ROBERT. High-risk obstetrics. Am. J. Obstet. \& Gynec. Estados Unidos. 105 (2): 241-247. Sep., 1969.

3. AUBRY, RICHARD, Y PENNINGTON, JOHN. Identification and evaluation of High-risk pregnancy: the perinatal concept. J. Clin. Obst. Gyn. Estados Unidos 16: 3-27, 1973.

4. BISHOP y FIENBERG, S. y HOLLAND, P. Discrete multivariate analysis. Estados Unidos. (Cambridge). Mit Press, 1975. análisis de factores y componentes principales que no permiten visualizar una estructura de factores.

\section{SUMMARY}

The study was done at the "Materno Infan til" Hospital in Bogotá D.E. in order to know the intrahospital obstetric risk factors. Four hundred eighty nine births were prospectively analized. Five risk factors (from 30 possible), significantly associated with harm/complication and relative risk greater than one, were chosen and ranked. The patients are tentatively classified according to high or low risk using two methods: complete multinomial and closest neighbor of $r=1$ order. An analysis is made of factors and main components wich do not permit visualizing a structure of factors.

5. COLIMON, Kahl. Fundamentos de Epidem iología. Medellín, Colimon, 1978.

6. COLOMBIA. MINISTERIO DE SALUD. UNIVERSIDAD JAVERIANA. UNIVERSIDAD DEL VALLE. Investigación de riesgo materno-infantil; estudio de riesgo reproductivo; segundo documento. Bogotá, 1976. 31p.

7. COLOMBIA. MINISTERIO DE SALUD. UNIVERSIDAD JAVERIANA. UNIVERSIDAD DEL VALLE. Investigación de riesgo materno-infantil; primer documento, Bogotá, 1976. 20p.

8. CONOVER, W.J. Practical nonparametric statistics. New York. Estados U nidos. Jon Wiley \& Sons Inc. 1971. 
9. DUARTE CONTRERAS, ALBERTO. ¿EI embarazo en adolescentes es siempre de alto riesgo? Rev. Col. Obst. y Gin. (Bogotá), 26 (6): 385-395, Nov. Dic. 1975.

10.. GLICK, N. Sample-based multinomial classification. Biometrics. Estados Unidos. 29, 241-256.

11. GRISWOLD, DON y CAVANAGH, DENIS. Prematurity - The epidemiologic profile of the "high-risk" mother. Am. J. Obst. \& Gynec. Estados Unidos. 96 (6): 878-882, Nov., 1966.

12. HILLS, M. Discrimination and allocation with discrete data. Applied Statistics, Estados Unidos, 16, 237-250. 1967.

13. HOBEL, CALVIN. Valoración del riesgo en medicina perinatal.

14. JUBIZ, ALFONSO et al. Determinaciónde la madurez fetal y neonatal en gestantes de alto riesgo, análisis citoquímico del líquido amniótico. Rev. Col. Obst. y Gin. (Bogotá), 28 (4): 137-147. Jul-Ago. 1977.

15. KARCHMER, SAMUEL Y CHAVEZ AZUELA, JOSE. Riesgo materno y fetal en cirugla obstétrica. Rev. Col. Obst. y Gin. (Bogotá), 22 (5): 297-314, Sept.Oct. 1971.

16. LINHART, H. Techiques for discriminant. Analysis with discrete variables. Estados Unidos. Metrica, 2: 138-149, 1959.

17. LACHENBRUCH, P. y GOLDSTEIN, M. Discriminant Analysis. Estados Uni: dos. 35, 69-85, Mar., 1979.
18. NESBITT, ROBERT, et al. Perinatal Mortality and morbidity. Am. J. O. Obst. \& Ginec. Estados Unidos. 93 (5): 702 719. Nov., 1965.

19. PASMIÑO DE OSORIO, SONIA. et. al: Embarazo de alto riesgo. Rev. Col. Obst. y Gin. (Bogotá), 29 (6): 264-301, Nov.Dic., 1978.

20. Plaut, RENATE. Análisis de riesgo; alcance y limitaciones para el administrador de salud. Boletín de la oficina sanitaria panamericana. Estados Unidos (Washing ton), abril., 1984.

21. QUEVEDO CAICEDO, JAIME. Un programa de salud pública para el niño con alto riesgo perinatal. Rev. Col. Obst. y Gin. (Bogotá), 29 (6): 302-303, Nov.Dic., 1978.

22. QUINTERO, CARLOS; RODRIGUEZ, JAIME y MUÑOZ, RAMIRO. Clínica de riesgo obstétrico. Rev. Col. Obst. y Gin. (Bogotá); 36 (1): 24-33, Enero-Feb., 1985.

23. SAS INSTITUTE STATISTICAL ANALYSIS SYSTEM. Sas User's guide. ed. Estados Unidos, (Cary), Sas institute, 1982.

24. SIEGEL, SIDNEY. Estadística no paramétrica. 2a. ed. México, Trillas, 1978.

25. SOKOL, ROBERT. et al. Clical application of high-risk scoring on an obstetric service. Am. J. Obstet \& Gynec. Estados Unidos. 128 (6): 652-661, Jul., 1977. 